\title{
DESENVOLVIMENTO DE UM ROTEIRO PARA MONITORAMENTO DO DESEMPENHO LOGÍSTICO EM EMPRESA DE SEMIJOIAS: um estudo de caso
}

\section{DEVELOPMENT OF A ROADMAP FOR MONITORING LOGISTICS PERFORMANCE IN A SEMI-JEWELRY COMPANY: a case study}

\author{
Fernando da Silva Coimbra ${ }^{\mathrm{I}}$ \\ Paulo Italo Jordão de Lucas ${ }^{\mathrm{II}}$ \\ Glaucia Aparecida Prates ${ }^{\text {III }}$ \\ Lesley Carina do Lago Attadia Galli ${ }^{\mathrm{IV}}$ \\ Antônio Francisco Savi ${ }^{\mathrm{V}}$
}

\begin{abstract}
RESUMO
Através de alguns indicadores logísticos, nomeadamente indicadores de entrega, transporte, stock e armazenagem. O objetivo deste artigo centra-se em analisar uma empresa de semijoias através de alguns indicadores logísticos, mediante a informação e dados que a empresa forneceu. Para o desenvolvimento desta análise, o método de pesquisa utilizado foi um caso de estudo sobre uma empresa de semijoias, empresa está situada no Brasil. Após a obtenção dos dados fornecidos pela empresa, os dados foram transferidos para uma folha em Excel e, posteriormente, analisados. Os resultados confirmam algumas relações entre os indicadores.
\end{abstract}

Palavras-chave: Indicadores. Logística. Entrega. Transporte. Stock.

\begin{abstract}
Through some logistical indicators, namely delivery, transport, stock, and storage indicators. The purpose of this article is to analyze a semi-jewelry company using some logistical indicators, using the information and data that the company provided. For the development of this analysis, the research method used was a case study about a semi-jewelry company, a company is located in Brazil. After obtaining the data provided by the company, the data were transferred to an Excel sheet and subsequently analyzed. The results confirm some relations between the indicators.
\end{abstract}

Keywords: Indicators. Logistics. Delivery. Transport. Stock.

Data de submissão do artigo: 28/06/2021.

Data de aprovação do artigo: 31/08/2021.

DOI: $10.33635 /$ sitefa.v4i1.173

\footnotetext{
${ }^{\text {I }}$ Engenheiro de produção - UFMS e Mestrando em Administração pela UNESP. E-mail: fernando.coimbra@unesp.br

II Engenheiro de produção - UFMS e Mestrando em Administração pela UNESP. E-mail: pauloitalo10@hotmail.com

III Doutora pela universidade estadual paulista UNESP.E-mail: glaucia@itapeva.unesp.br

IV Doutora pela universidade estadual paulista UNESP. E-mail: lesley.attadia@unesp.br

v Doutor pela universidade estadual paulista UNESP. E-mail: antonio.savi@unesp.br
} 


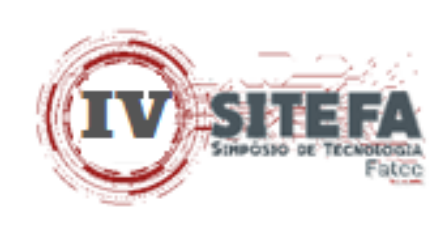

\section{INTRODUÇÃO}

O segmento de joias, semi joias e bijuterias apresentam espaço relevante no cenário mundial, de acordo com Dauriz, Remy e Tochtermann (2014) no ano de 2014 as vendas do setor representaram um montante de US\$ 148 bilhões. Em 2018 o Brasil ocupava a $12^{\mathrm{a}}$ posição no mercado mundial no setor, com incremento de $9 \%$ em relação ao ano anterior. No que tange ao mercado interno as vendas atingiram $\mathrm{R} \$ 12,6$ bilhões, e até 2023, espera-se que este número ultrapasse R\$ 13 bilhões (IBGM, 2019).

Destaca-se que o setor tem a presença de alguns grandes players, no entanto também é composto por pequenas e médias empresas com alcance regional. Nesse ponto, destaca-se que este estudo abrange uma dessas empresas, classificadas como uma empresa de pequeno porte. Que de acordo com Olave e Amato Neto (2001), tradicionalmente são empresas de grande importância por sua capacidade de gerar empregos ou pela contribuição para a produção de bens e oferta de serviços.

De acordo com Chiavenato (2007), é cada vez mais necessário que as empresas busquem melhorias nos processos, desenvolvam e inovem seus produtos e serviços ofertados, além de aprimorar suas estratégias para ter vantagem competitiva em relação aos seus concorrentes.

Nesse ponto, destaca-se que a logística é um importante pilar para melhoria do desempenho operacional e agrega valor ao cliente, considerando que, dentre outras coisas, é responsável pela entrega dos produtos. Segundo Carvalho (2017), logística é um processo de planejamento, implementação e controle dos fluxos de materiais, serviços e informações, do ponto de origem ao ponto de consumo.

Como ferramenta para avaliação da performance logística tem-se os indicadores de desempenho, que de forma genérica, segundo a Fundação Nacional da Qualidade - FNQ (2020), geram informações quantitativas ou qualitativas que expressam a performance de um determinado processo, permitindo acompanhar sua evolução ao longo do tempo e compará-lo com outras organizações.

Dessa forma, e considerando as implicações gerenciais, evidencia-se o seguinte problema de pesquisa: como desenvolver um roteiro para monitoramento do desempenho logístico em uma empresa de semijoias, para fornecer subsídios para o gerenciamento da empresa?

Neste contexto, o objetivo deste trabalho foi desenvolver um roteiro para monitoramento do desempenho logístico, em uma empresa de pequeno porte, no ramo de semijoias, que atua no Centro-Oeste do Brasil, para fornecer informações que contribua, de forma estruturada, com o gerenciamento das operações da empresa.

Para alcançar o objetivo geral foram definidos os seguintes objetivos específicos: a) selecionar os indicadores de desempenho logístico mais adequados às características da empresa estudada; b) levantar as informações necessárias e calcular os indicadores selecionados; c) avaliar a empresa pela ótica do desempenho logístico; d) propor rotinas de utilização dos indicadores para contribuir com o gerenciamento da empresa.

Os resultados do estudo de caso demonstram uma maneira de monitorar o desempenho logístico em uma empresa de semijoias, de forma que seja capaz de fornecer informações gerenciais para tomada de decisão. Destaca-se que os indicadores logísticos fornecem subsídios para o gerenciamento da empresa, permitindo um processo mais estruturado de gestão das operações. 


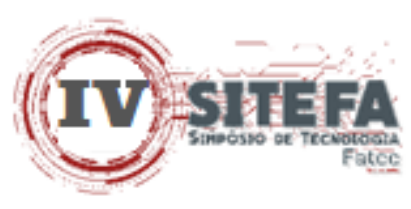

Para a apresentação do presente trabalho, este artigo foi estruturado em mais quatro seções subsequentes. Na seção 2 é apresentada a fundamentação teórica, que aborda sobre a logística e a cadeia de suprimentos, indicadores de desempenho e indicadores de desempenho logísticos. Na seção 3 apresenta-se o método de pesquisa utilizado, o estudo de caso. Na seção 4 é desenvolvido o estudo, assim como a discussão dos resultados. Por fim, na seção 5 são tecidas as considerações finais.

\section{LOGÍSTICA}

A logística é “[...] o processo de planejamento, implementação e controle eficiente e eficaz do fluxo de armazenagem de mercadorias em processo, produtos terminados e fluxo de informações, desde o ponto de origem até o ponto de consumo, com o objetivo de atender às necessidades do cliente" (COUNCIL OF LOGISTICS MANAGEMENT, 1986). Tendo em vista a definição acima, a logística se baseia em fluxos de produtos e informações, gerando uma cadeia de movimentação ininterrupta para a otimização dos processos, agregando-lhes valor durante todo o processo de transformação do bem ou serviço.

Figura 1 - Processo de transformação

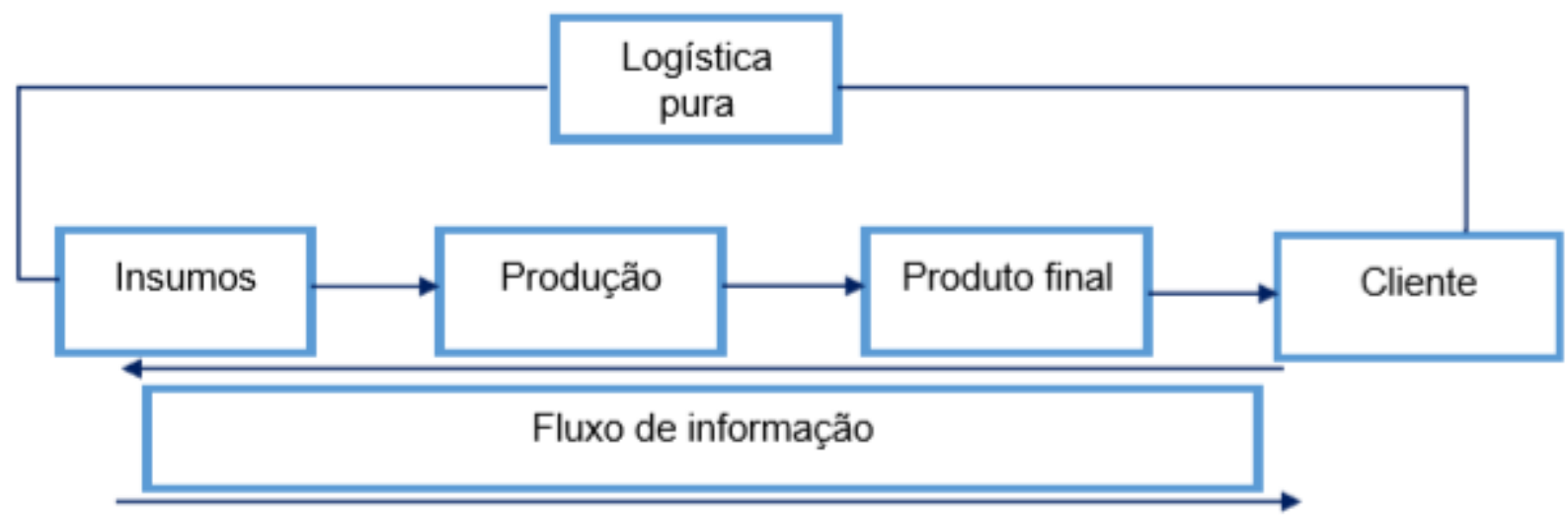

Fonte: os autores, 2021

\subsection{Cadeia de suprimentos}

De acordo com os membros do Council of supply chain management professionals (CSCMP) definem a logística de suprimentos como" [...] integração de processos de negócios-chave desde o último usuário até o fornecedor de origem que abastece produtos, serviços e informações que adicionam valor para o cliente e outros stakeholders' (AZEVEDO et al, 2011, p.4). O que diferencia a logística da cadeia de suprimentos é a integração de processos dentro e fora da empresa, considerando todos os elos, desde fornecedores, intermediários, movimentadores etc. Uma característica para este modelo, é que se deve é verificar o processo-chave e criar um valor conjunto para todas as partes da cadeia de suprimentos, isso pode gerar vantagem competitiva. 


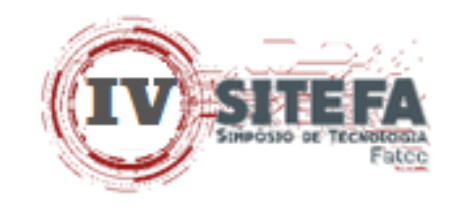

Figura 2 - Oito processos chave

Processos de Negócios e Agentes da Cadeia de Suprimentos

\section{Fluxo de Informaçōes}

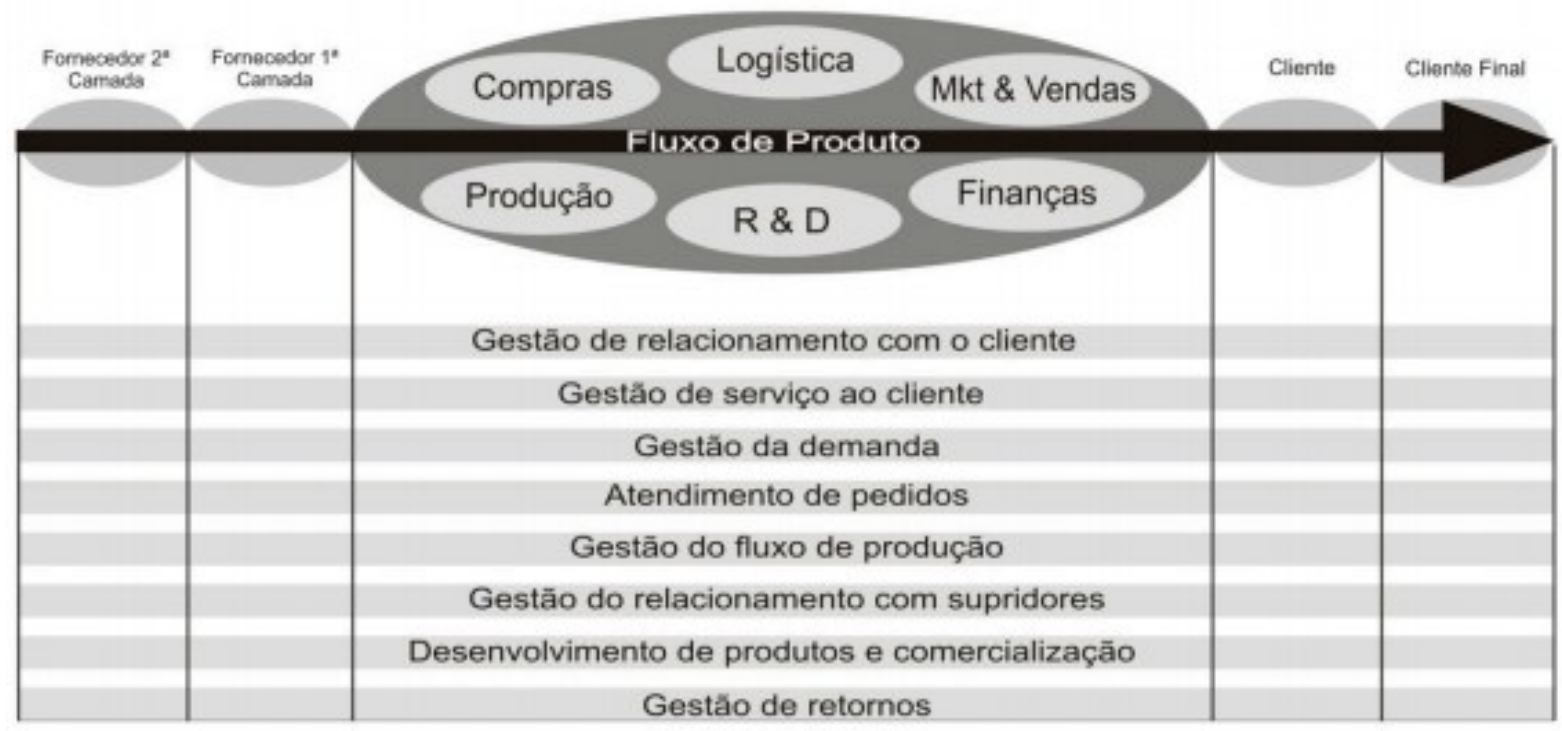

Fonte: Adaptado de Croxton, Garcia-Dasturge e Lambert (2001, p. 14)

Na figura 2, vemos os oito, processos-chave e os seis departamentos, que ocorrem ao longo de toda a cadeia de suprimentos, desde o ponto de origem até o consumidor final, isto também depende do segmento que a empresa trabalha.

\subsection{Indicadores de desempenho}

Em uma organização deve-se quantificar variáveis de interesse e objetivo da empresa; o desempenho, possui a ideia intrínseca de que algo já foi finalizado, exercido. Compreender a realidade da empresa, permitindo melhores decisões durante os processos produtivos acarretará melhores ações para otimizações substanciais na organização.

Segundo Bandeira (1997, p. 111), "medir o desempenho, de fato, somente se justifica quando existe o objetivo de aperfeiçoá-lo". Ideia central para implantação de indicadores de desempenho, sua operacionalização ocorre através de medições que buscam dimensionar o ato, processo ou atividades, que se deseja quantificar, as informações contidas numa operação são valiosas e saber separá-las de forma concisa torna as operações mais enxutas e eficazes.

De fato, a crescente mudança no cenário mundial acaba exigindo cada dia mais das empresas, pois a melhoria dos processos operacionais deve ser medida numa rotina constante, com o intuito de gerar eficiência, Para Neely et al. (1995, p. 80), eficiência é o quanto se utiliza economicamente dos recursos alocados, visando atingir um determinado nível de satisfação.

\subsection{Indicadores de desempenho logístico}

Segundo Conceição e Quintão (2004), os indicadores de desempenho são utilizados para mensurar, como e de que maneira, os elos da cadeia de suprimentos contribuem para a 


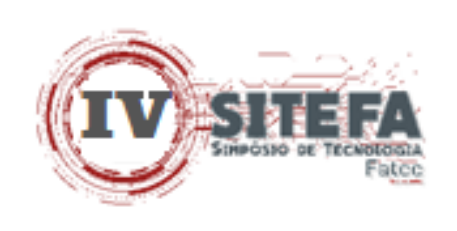

gestão e integração. Diante do explanado, as formas de supervisionar os elos da cadeia, dependem de como a empresa funciona.

De acordo com Ballou (2001) as atividades logísticas devem ser encaradas como processo contínuo, cujo desempenho, deve ser monitorado. De fato, à medida que a competência logística se torna um fator mais crítico na criação e na manutenção da vantagem competitiva, a precisão nessas tarefas torna-se mais importante, pois a diferença entre operações rentáveis e não rentáveis cada vez está menor.

\section{METODOLOGIA DA PESQUISA}

O método de pesquisa utilizado foi o estudo de caso, o qual permite identificar a cadeia de suprimentos e as operações logísticas da empresa, selecionar os indicadores logísticos que mais se adequam às operações da empresa de semijoias e calcular e interpretar os indicadores logísticos para identificar os pontos positivos e sugerir oportunidades para o desempenho logístico da empresa.

A abordagem utilizada foi predominantemente qualitativa, que segundo Llewellyn e Northcott (2007) é focada na identificação das características de eventos e organizações. Quanto ao número de objetos estudados, a escolha de um único caso objetivou a profundidade do estudo, pois de acordo com Freitas e Jabbour (2011), o estudo de um único caso é o método mais adequado para conhecer com profundidade todas as variações de um determinado fenômeno organizacional.

O estudo de caso é uma investigação empírica que investiga um fenômeno contemporâneo dentro de seu contexto da vida real, especialmente quando os limites entre o fenômeno e o contexto não estão claramente definidos. Este método baseia-se em várias fontes de evidências e beneficia-se do desenvolvimento prévio de proposições teóricas para conduzir a coleta e a análise de dados (YIN, 2014).

A escolha desse método de pesquisa foi motivada pelo fato do estudo de caso apresentar vantagens em relação à outras estratégias de pesquisa, destacando sua capacidade de lidar com uma ampla variedade de evidências, o que é fundamental para desenvolver um roteiro para monitoramento do desempenho logístico de uma empresa.

De acordo com Ganga (2012) para o desenvolvimento desse método de pesquisa devem ser claramente definidos: a) propósito do estudo; b) coleta de dados; c) horizonte de pesquisa; e d) análise de dados.

No que se refere ao propósito, destaca-se que este estudo apresenta características descritivas, as quais segundo Vergara (2004) expõe características de determinado fenômeno, podendo também estabelecer correlações entre variáveis e definir sua natureza.

No que tange à coleta de dados, Voss, Tsikriktsis e Frohlich (2002) afirmam que no método de estudo de caso utiliza-se de uma combinação de diferentes instrumentos para estudar um mesmo fenômeno, tais como: observação direta do pesquisador, questionamentos aos envolvidos; e interpretação dos dados operacionais. Neste trabalho, os instrumentos de pesquisa utilizados foram: entrevistas e análises de documentos.

As entrevistas foram realizadas com o proprietário da empresa de semijoias, tiveram caráter semiestruturado, em que as principais perguntas tiveram o objetivo: a) conhecer a empresa e as características de mercado; b) identificar as operações logísticas da empresa em seus quatro pilares (estoque, entrega, transporte e armazenagem); e c) levantar os dados para cálculo dos indicadores logísticos. Além disso, com o intuito de coletar informações para o 


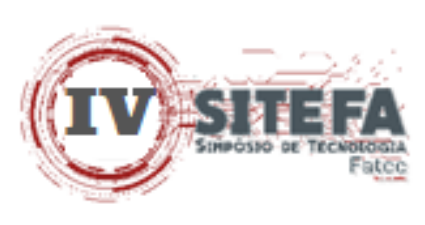

cálculo quantitativo dos indicadores logísticos, foram analisados os registros e históricos da empresa, que ficam contidos em um sistema de cunho gerencial interno.

Sobre o horizonte de tempo, o estudo de caso desenvolvido foi classificado como cross-section ou transversal, sendo realizado em aproximadamente duas semanas. Por fim, em relação à análise de dados, tem-se que a mesma foi dividida entre: a) definição dos indicadores logísticos; b) cálculo e interpretação dos indicadores logísticos da empresa de semijoias.

\section{RESULTADOS E DISCUSSÃO}

A toque de requinte é uma empresa no ramo de semi joia, iniciada em 2016 a partir de uma necessidade dos fundadores de ter uma renda para as necessidades diárias. De acordo com a aceitação das clientes próximas, visualizaram uma oportunidade de expansão para repassar a outras pessoas interessadas em revender e ter uma renda extra. Hoje atua no leste de mato grosso do sul e oeste paulista, distribuindo sua mercadoria para vários pontos comerciais e pessoas físicas. A sua missão é satisfazer os clientes com qualidade e pontualidade. A sua visão é tornar-se um ícone em inovação de peças contemporâneas. Os seus valores: integridade, comprometimento e honestidade.

\subsection{Indicador: pedido perfeito}

O índice de pedido perfeito mede a percentagem de pedidos entregues no prazo estipulado com o cliente, completo, sem avarias e sem problemas na documentação fiscal. Através da tabela $1 \mathrm{E}$ de modo geral, a percentagem de pedidos perfeitos variam entre os $65 \%$ e $97 \%$. A percentagem mais baixa foi obtida em janeiro de 2020 com 65,66\% e a mais alta foi atingida em junho de 2020 com $96,26 \%$.

Tabela 1 - Pedido perfeito 2019/20

\begin{tabular}{|c|c|c|c|c|c|c|c|}
\hline \multicolumn{4}{|c|}{ Pedido Perfeito - 2019} & \multicolumn{4}{|c|}{ Pedido Perfeito - 2020} \\
\hline & $\begin{array}{c}\mathbf{N}^{0} \text { Pedidos } \\
\text { perfeitos } \\
\text { entregues }\end{array}$ & $\begin{array}{l}\text { Total de } \\
\text { pedidos } \\
\text { expedidos }\end{array}$ & PP & & $\begin{array}{l}\mathbf{N}^{0} \text { Pedidos } \\
\text { perfeitos } \\
\text { entregues }\end{array}$ & $\begin{array}{l}\text { Total de } \\
\text { pedidos } \\
\text { expedidos }\end{array}$ & PP \\
\hline jan./19 & 140 & 180 & $77,78 \%$ & jan./20 & 130 & 198 & $65,66 \%$ \\
\hline fev./19 & 120 & 160 & $75,00 \%$ & fev./20 & 120 & 168 & $71,43 \%$ \\
\hline $\operatorname{mar} / 19$ & 130 & 163 & $79,75 \%$ & $\operatorname{mar} / 20$ & 124 & 170 & $72,94 \%$ \\
\hline abr./19 & 145 & 175 & $82,86 \%$ & abr./20 & 125 & 185 & $67,57 \%$ \\
\hline mai/19 & 160 & 180 & $88,89 \%$ & $\mathrm{mai} / 20$ & 150 & 194 & $77,32 \%$ \\
\hline jun./19 & 170 & 182 & $93,41 \%$ & jun./20 & 180 & 187 & $96,26 \%$ \\
\hline jul./19 & 165 & 190 & $86,84 \%$ & jul./20 & 185 & 195 & $94,87 \%$ \\
\hline ago./19 & 176 & 195 & $90,26 \%$ & ago./20 & 180 & 210 & $85,71 \%$ \\
\hline set/19 & 160 & 190 & $84,21 \%$ & set/20 & 170 & 200 & $85,00 \%$ \\
\hline out $/ 19$ & 170 & 200 & $85,00 \%$ & out $/ \mathbf{2 0}$ & 166 & 190 & $87,37 \%$ \\
\hline nov./19 & 178 & 210 & $84,76 \%$ & nov./20 & 180 & 200 & $90,00 \%$ \\
\hline $\operatorname{dez} / 19$ & 190 & 230 & $82,61 \%$ & $\operatorname{dez} / 20$ & 200 & 210 & $95,24 \%$ \\
\hline
\end{tabular}




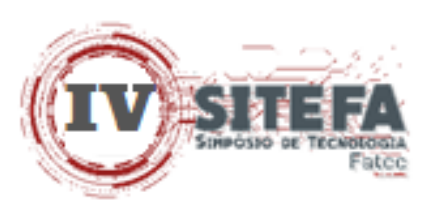

Pode-se concluir que do ano 2019 para 2020 existiu um aumento da percentagem do pedido perfeito pois, comparativamente ao ano anterior, 2020 teve uma percentagem mais alta de pedidos perfeito. (Gráfico 1)

\section{Gráfico 1 - Pedidos perfeitos}

\section{Pedidos Perfeitos}

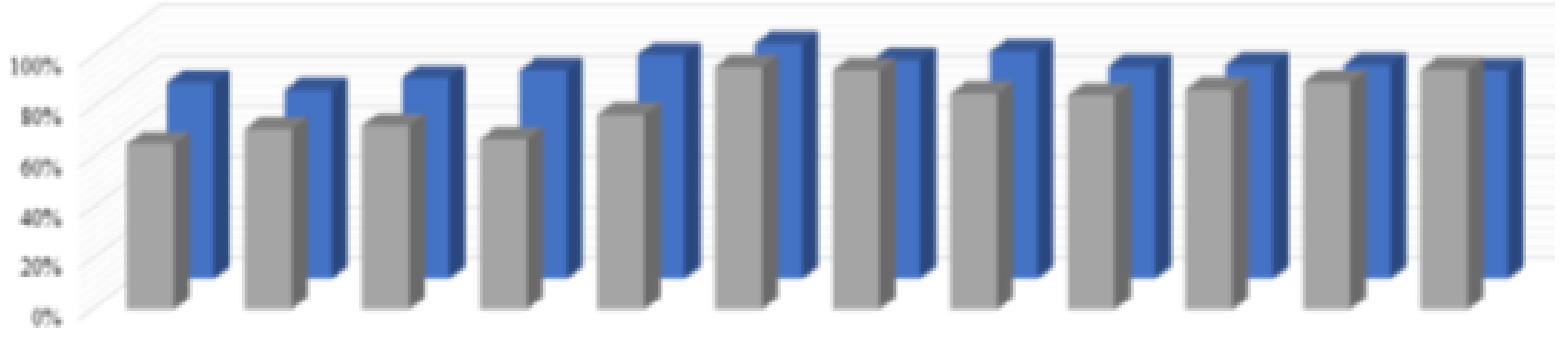

Fonte: os autores, 2021

E Redido Perfecto - $2020 \quad$ Inedido Perfects - 2019

\section{2 Índice de porcentagem de entrega}

O índice de porcentagem de entregas no prazo, ou seja, sem atrasos. A verificação deste indicador permite identificar possíveis problemas relacionados ao tempo de transporte. Uma análise que pode ser feita é os possíveis problemas que podem estar ocorrendo no tempo de transporte.

De acordo com a tabela 2, pode-se verificar que existe uma oscilação nos números durante o período de 12 meses. Fator pelo qual se dá a necessidade de a empresa levar o pedido a locais que variam de acordo com o ambiente, ou seja, malha asfáltica, tempo chuvoso, muita poeira etc.

Tabela 2 - \% de entrega 2019/20

\begin{tabular}{|c|c|c|c|c|c|c|c|}
\hline \multicolumn{4}{|c|}{$\%$ de entregas no prazo - 2019} & \multicolumn{4}{|c|}{$\%$ de entregas no prazo - 2020} \\
\hline & $\begin{array}{l}\mathrm{N}^{0} \text { entregas } \\
\text { realizadas no } \\
\text { prazo }\end{array}$ & $\begin{array}{l}\text { Total entregas } \\
\text { realizadas }\end{array}$ & $\%$ ERP & & $\begin{array}{l}\mathrm{N}^{0} \text { entregas } \\
\text { realizadas no } \\
\text { prazo } \\
\end{array}$ & $\begin{array}{l}\text { Total entregas } \\
\text { realizadas }\end{array}$ & $\%$ ERP \\
\hline Jan & 120 & 175 & $69 \%$ & Jan & 130 & 180 & $72 \%$ \\
\hline Fev. & 125 & 165 & $76 \%$ & Fev. & 120 & 160 & $75 \%$ \\
\hline Mar & 124 & 150 & $83 \%$ & Mar & 124 & 163 & $76 \%$ \\
\hline Abr. & 120 & 175 & $69 \%$ & Abr. & 125 & 175 & $71 \%$ \\
\hline Mai & 140 & 175 & $80 \%$ & Mai & 150 & 180 & $83 \%$ \\
\hline Jun. & 170 & 180 & $94 \%$ & Jun. & 180 & 182 & $99 \%$ \\
\hline Jul. & 180 & 187 & $96 \%$ & Jul. & 185 & 190 & $97 \%$ \\
\hline Ago. & 174 & 190 & $92 \%$ & Ago. & 180 & 195 & $92 \%$ \\
\hline Set & 175 & 195 & $90 \%$ & Set & 170 & 190 & $89 \%$ \\
\hline Out & 170 & 205 & $83 \%$ & Out & 166 & 190 & $87 \%$ \\
\hline Nov. & 170 & 210 & $81 \%$ & Nov. & 180 & 210 & $86 \%$ \\
\hline Dez & 200 & 230 & $87 \%$ & Dez & 200 & 210 & $95 \%$ \\
\hline
\end{tabular}

Fonte: os autores, 2021

Visto de no gráfico 2, pode-se observar que os meses de abril e maio de 2019, mesmo o total de entregas sendo o mesmo, teve-se uma variação de $11 \%$ na taxa de porcentagem de 


\section{IVITEFA}

Protec

entregas no prazo. Entretanto os meses de setembro e outubro tiveram, praticamente, um mesmo índice, variando apenas $2 \%$, considerando que a quantidade de entregas realizadas foi o mesmo.

Gráfico 2 - \% de entregas no prazo.

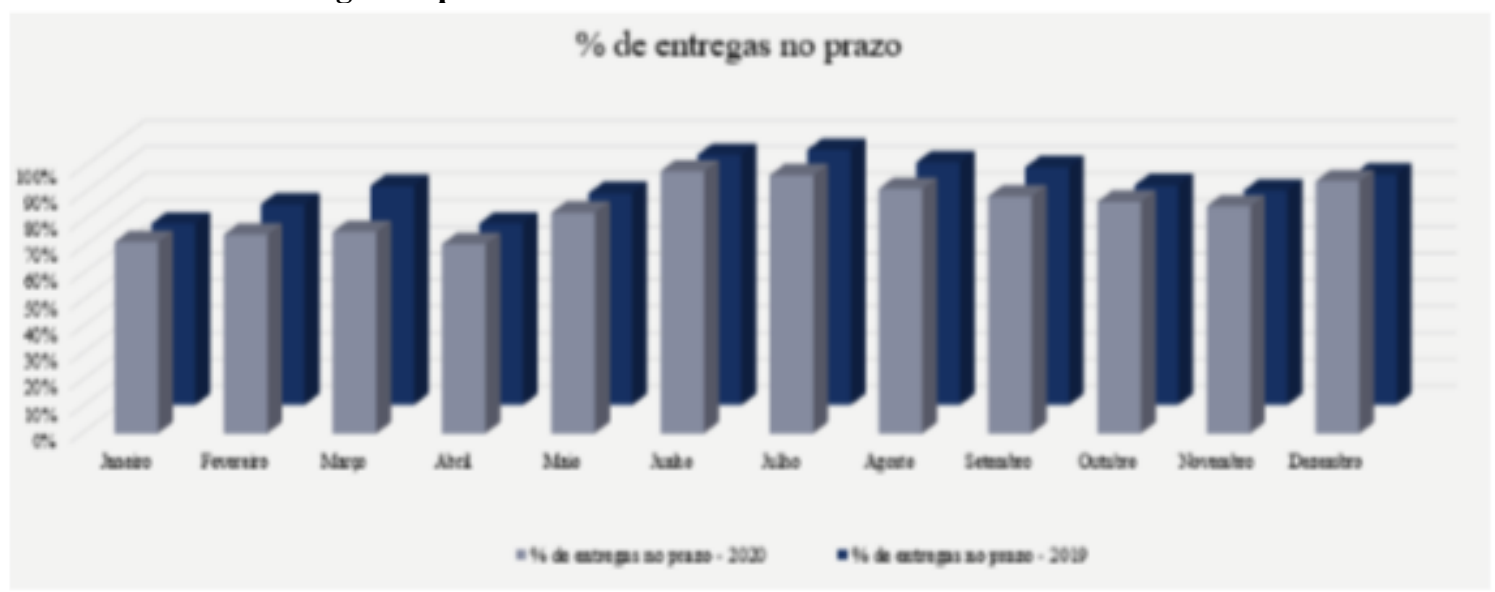

Fonte: os autores, 2021

\section{3 Índice de atendimento do pedido}

O índice de atendimento do pedido mede a percentagem de pedidos atendidos na sua totalidade e na diversidade e itens no primeiro envio ao cliente. As percentagens desde indicador variam entre $70 \%$ e $99 \%$, sendo que o ano 2019 apresentou valores mais positivos em relação ao ano de 2020 .

Tabela 3 - Índice de atendimento de pedido

\begin{tabular}{|c|c|c|c|c|c|c|c|}
\hline \multicolumn{4}{|c|}{ Índice de Atendimento do Pedido - 2019} & \multicolumn{4}{|c|}{ Índice de Atendimento do Pedido - 2020} \\
\hline & $\begin{array}{l}\mathrm{N}^{0} \text { pedidos } \\
\text { atendidos na } \\
\text { totalidade }\end{array}$ & $\begin{array}{l}\text { Total de } \\
\text { pedidos } \\
\text { expedidos }\end{array}$ & OFR & & $\begin{array}{l}\mathrm{N}^{0} \text { pedidos } \\
\text { atendidos na } \\
\text { totalidade }\end{array}$ & $\begin{array}{l}\text { Total de } \\
\text { pedidos } \\
\text { expedidos }\end{array}$ & OFR \\
\hline Jan & 150 & 180 & $83 \%$ & Jan & 140 & 198 & $71 \%$ \\
\hline Fev. & 130 & 160 & $81 \%$ & Fev. & 130 & 168 & $77 \%$ \\
\hline Mar & 135 & 163 & $83 \%$ & Mar & 135 & 170 & $79 \%$ \\
\hline Abr. & 165 & 175 & $94 \%$ & Abr. & 136 & 185 & $74 \%$ \\
\hline Mai & 170 & 180 & $94 \%$ & Mai & 150 & 194 & $77 \%$ \\
\hline Jun. & 178 & 182 & $98 \%$ & Jun. & 190 & 187 & $102 \%$ \\
\hline Jul. & 168 & 190 & $88 \%$ & Jul. & 190 & 195 & $97 \%$ \\
\hline Ago. & 179 & 195 & $92 \%$ & Ago. & 185 & 210 & $88 \%$ \\
\hline Set & 165 & 190 & $87 \%$ & Set & 175 & 200 & $88 \%$ \\
\hline Out & 180 & 200 & $90 \%$ & Out & 170 & 190 & $89 \%$ \\
\hline Nov. & 190 & 210 & $90 \%$ & Nov. & 180 & 200 & $90 \%$ \\
\hline Dez & 195 & 230 & $85 \%$ & Dez & 205 & 210 & $98 \%$ \\
\hline
\end{tabular}

Fonte: os autores, 2021

O gráfico 3 realça a diferença e as várias oscilações ao longo dos 2 anos, 2019 e 2020 respetivamente. Apesar de 2020 ter apresentado valores mais baixos, os meses de junho, julho, setembro e dezembro conseguiram apresentar valores superiores a 2019. 
Gráfico 3 - Índice de atendimento do pedido

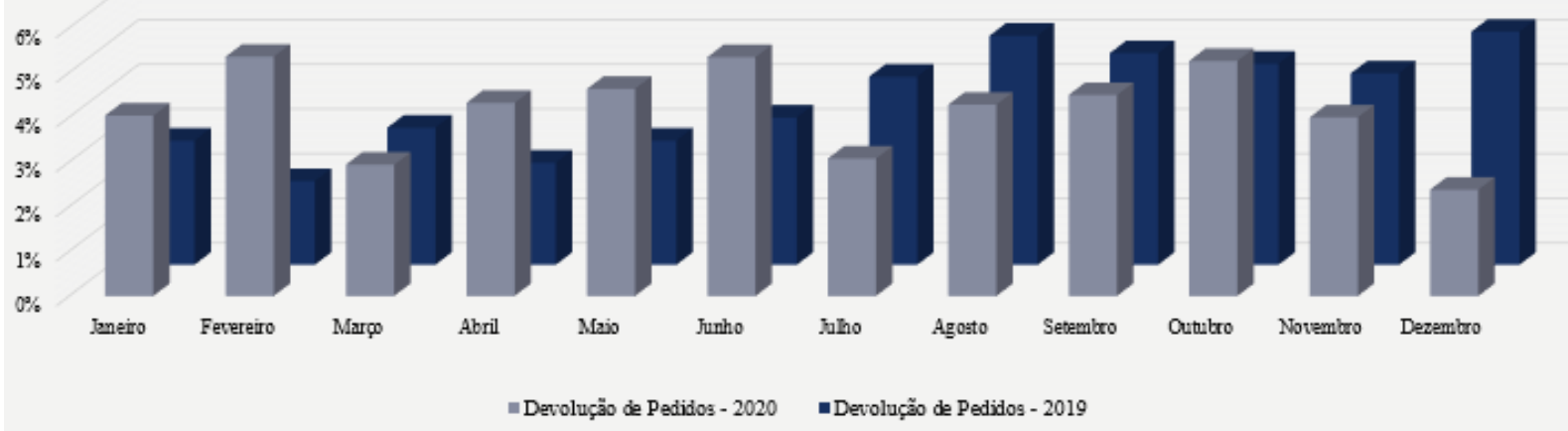

Fonte: os autores, 2021

\subsection{Devolução de pedidos}

O índice de pedidos mede as devoluções das entregas realizadas. Através da tabela 4 , pode-se concluir que as percentagens de ambos os anos variam entre $1 \%$ e $6 \%$, atingindo a percentagem mais alta em fevereiro de 2020 com $5,36 \%$ e a percentagem mais baixa em fevereiro de 2019 com 1,88\%.

Tabela 4 - Devolução de pedidos

\begin{tabular}{|c|c|c|c|c|c|c|c|}
\hline \multicolumn{4}{|c|}{ Devolução de Pedidos - 2019} & \multicolumn{4}{|c|}{ Devolução de Pedidos - 2020} \\
\hline & $\begin{array}{c}\text { Total } \\
\text { devolvido }\end{array}$ & Total enviado & $\begin{array}{l}\text { Devolução } \\
\text { de pedidos }\end{array}$ & & $\begin{array}{c}\text { Total } \\
\text { devolvido }\end{array}$ & $\begin{array}{c}\text { Total } \\
\text { enviado }\end{array}$ & $\begin{array}{l}\text { Devolução } \\
\text { de pedidos }\end{array}$ \\
\hline Jan & 5 & 180 & $3 \%$ & Jan & 8 & 198 & $4 \%$ \\
\hline Fev. & 3 & 160 & $2 \%$ & Fev. & 9 & 168 & $5 \%$ \\
\hline Mar & 5 & 163 & $3 \%$ & Mar & 5 & 170 & $3 \%$ \\
\hline Abr. & 4 & 175 & $2 \%$ & Abr. & 8 & 185 & $4 \%$ \\
\hline Mai & 5 & 180 & $3 \%$ & Mai & 9 & 194 & $5 \%$ \\
\hline Jun. & 6 & 182 & $3 \%$ & Jun. & 10 & 187 & $5 \%$ \\
\hline Jul. & 8 & 190 & $4 \%$ & Jul. & 6 & 195 & $3 \%$ \\
\hline Ago. & 10 & 195 & $5 \%$ & Ago. & 9 & 210 & $4 \%$ \\
\hline Set & 9 & 190 & $5 \%$ & Set & 9 & 200 & $5 \%$ \\
\hline Out & 9 & 200 & $5 \%$ & Out & 10 & 190 & $5 \%$ \\
\hline Nov. & 9 & 210 & $4 \%$ & Nov. & 8 & 200 & $4 \%$ \\
\hline Dez & 12 & 230 & $5 \%$ & Dez & 5 & 210 & $2 \%$ \\
\hline
\end{tabular}

Fonte: os autores, 2021

Com o gráfico 4, conclui-se que aumentaram as devoluções no início de 2020, tendo baixado no segundo semestre do mesmo ano, equilibrando a balança dos dois anos em análise. 


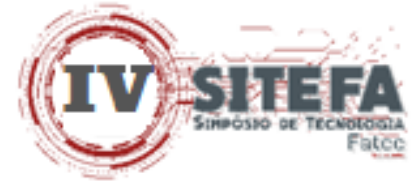

Gráfico 4 - Devolução de pedidos

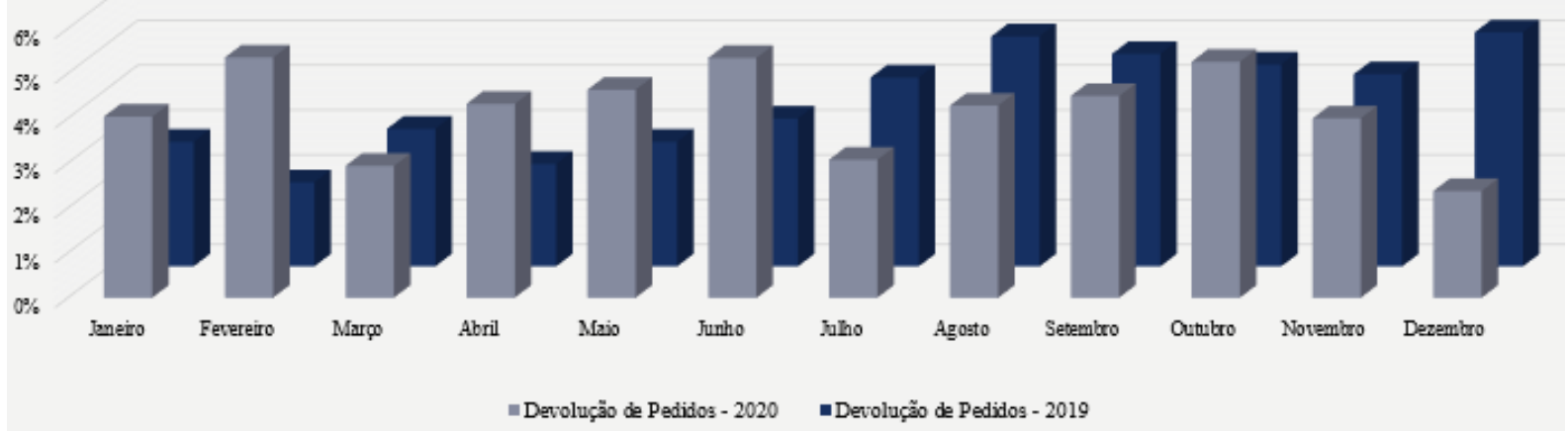

Fonte: os autores, 2021

\subsection{Custo de transporte}

O custo de transporte são todas as despesas, fixas e variáveis, realizadas na movimentação do produto desde a origem até seu destino. Existem várias variáveis que devem ser levadas em consideração quando se mede este indicador, pois a oscilação de combustível, por exemplo, nos locais de rota do veículo que levará este produto pode mudar. De acordo com a tabela 5, tem-se os valores do ano de 2019 e 2020 demonstrando os valores dos custos de transporte.

Tabela 5 - Custo de transporte

\section{Custo de transporte - 2019}

\section{Custo total}

de

transporte

$\begin{array}{cc}\begin{array}{c}\text { Receita de } \\ \text { vendas }\end{array} & \begin{array}{c}\text { Custo de } \\ \text { transporte }\end{array}\end{array}$

Custo de transporte -2020

$\begin{array}{ccc}\text { Custo total } & \begin{array}{c}\text { Receita de } \\ \text { vendas }\end{array} & \begin{array}{c}\text { Custo de } \\ \text { transporte }\end{array}\end{array}$

\begin{tabular}{lllllllllll}
\hline Jan & $\mathrm{R} \$$ & 285,00 & $\mathrm{R} \$$ & $12.000,00$ & $2,38 \%$ & Jan & $\mathrm{R} \$$ & 350,00 & $\mathrm{R} \$ 15.000,00$ & $2,33 \%$ \\
Fev. & $\mathrm{R} \$$ & 290,00 & $\mathrm{R} \$$ & $12.340,00$ & $2,35 \%$ & Fev. & $\mathrm{R} \$$ & 300,00 & $\mathrm{R} \$ 14.350,00$ & $2,09 \%$ \\
Mar & $\mathrm{R} \$$ & 280,00 & $\mathrm{R} \$$ & $13.000,00$ & $2,15 \%$ & Mar & $\mathrm{R} \$$ & 310,00 & $\mathrm{R} \$ 14.735,00$ & $2,10 \%$ \\
Abr. & $\mathrm{R} \$$ & 310,00 & $\mathrm{R} \$$ & $12.950,00$ & $2,39 \%$ & Abr. & $\mathrm{R} \$$ & 330,00 & $\mathrm{R} \$ 14.900,00$ & $2,21 \%$ \\
Mai & $\mathrm{R} \$$ & 300,00 & $\mathrm{R} \$$ & $10.000,00$ & $3,00 \%$ & Mai & $\mathrm{R} \$$ & 380,00 & $\mathrm{R} \$ 15.154,00$ & $2,51 \%$ \\
Jun. & $\mathrm{R} \$$ & 290,00 & $\mathrm{R} \$$ & $12.000,00$ & $2,42 \%$ & Jun. & $\mathrm{R} \$$ & 390,00 & $\mathrm{R} \$ 15.250,00$ & $2,56 \%$ \\
Jul. & $\mathrm{R} \$$ & 310,00 & $\mathrm{R} \$$ & $13.000,00$ & $2,38 \%$ & Jul. & $\mathrm{R} \$$ & 400,00 & $\mathrm{R} \$ 15.650,00$ & $2,56 \%$ \\
Ago. & $\mathrm{R} \$$ & 300,00 & $\mathrm{R} \$$ & $13.235,00$ & $2,27 \%$ & Ago. & $\mathrm{R} \$$ & 405,00 & $\mathrm{R} \$ 15.780,00$ & $2,57 \%$ \\
Set & $\mathrm{R} \$$ & 320,00 & $\mathrm{R} \$$ & $13.475,00$ & $2,37 \%$ & Set & $\mathrm{R} \$$ & 420,00 & $\mathrm{R} \$ 15.600,00$ & $2,69 \%$ \\
Out & $\mathrm{R} \$$ & 310,00 & $\mathrm{R} \$$ & $14.100,00$ & $2,20 \%$ & Out & $\mathrm{R} \$$ & 448,00 & $\mathrm{R} \$ 16.110,00$ & $2,78 \%$ \\
Nov. & $\mathrm{R} \$$ & 310,00 & $\mathrm{R} \$$ & $14.230,00$ & $2,18 \%$ & Nov. & $\mathrm{R} \$$ & 450,00 & $\mathrm{R} \$ 16.925,00$ & $2,66 \%$ \\
Dez & $\mathrm{R} \$$ & 320,00 & $\mathrm{R} \$$ & $14.290,00$ & $2,24 \%$ & Dez & $\mathrm{R} \$$ & 440,00 & $\mathrm{R} \$ 17.200,00$ & $2,56 \%$ \\
\hline
\end{tabular}

Fonte: os autores, 2021

Pode-se perceber, mesmo em alguns meses o valor do custo total de transporte sendo maior de 2020 para 2019, exemplo mês de fevereiro, o indicador custo de transporte ficou menor. Isso demonstra que a receita de venda influencia diretamente no comparativo mês a mês para esse KPI. Em análise do gráfico 5, percebe-se que o mês de maio teve uma taxa de $3 \%$ em 2019 para $2,51 \%$ em relação a 2020. 


\section{(IV) SITEFA}

Gráfico 5 - Custo de transporte

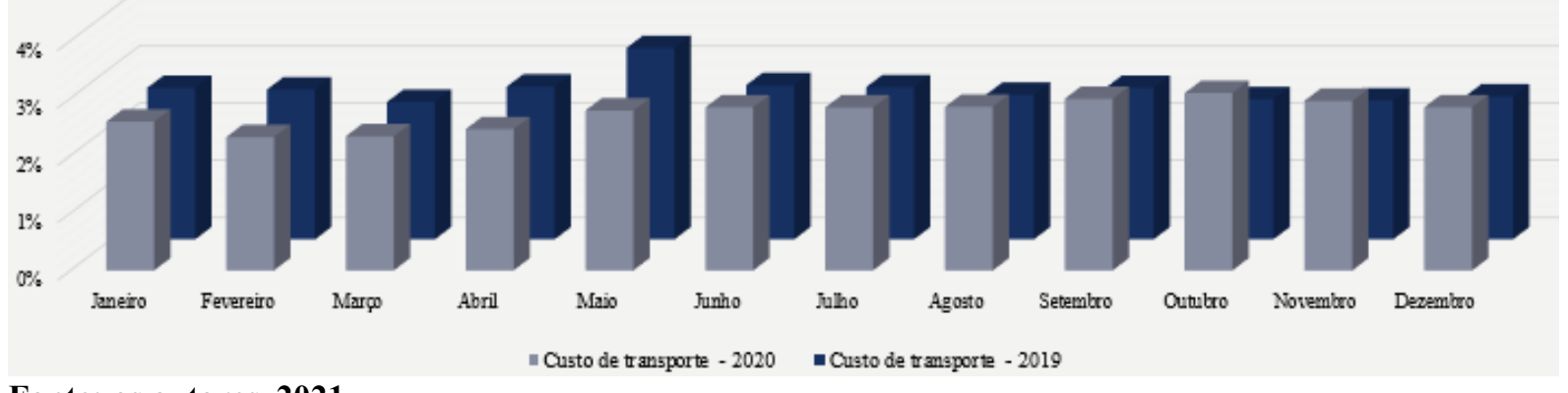

Fonte: os autores, 2021

\subsection{Cobertura de Stock}

A cobertura de stock mede o tempo em que o stock existente é suficiente para atender a procura, sem a necessidade de reposição, ou seja, indica quantos dias ou semanas de stock temos no armazém. A tabela 6 apresenta os valores mensais do ano de 2019 e 2020 relativamente à empresa Toque de Requinte - Semi Joias.

Tabela 6 - Cobertura de Stock

\begin{tabular}{|c|c|c|c|c|c|c|c|}
\hline \multicolumn{4}{|c|}{ Cobertura de Stock - 2019} & \multicolumn{4}{|c|}{ Cobertura de Stock - 2020} \\
\hline & $\begin{array}{l}\text { Stock } \\
\text { atual }\end{array}$ & $\begin{array}{c}\text { Previsão de } \\
\text { venda mensal }\end{array}$ & $\begin{array}{c}\text { Cobertura de } \\
\text { Stock }\end{array}$ & & $\begin{array}{l}\text { Stock } \\
\text { atual }\end{array}$ & $\begin{array}{c}\text { Previsão de } \\
\text { venda mensal }\end{array}$ & $\begin{array}{c}\text { Cobertura de } \\
\text { Stock }\end{array}$ \\
\hline Jan & 170 & 190 & 3,83 & Jan & 100 & 200 & 2,14 \\
\hline Fev. & 165 & 170 & 4,15 & Fev. & 80 & 180 & 1,90 \\
\hline Mar & 170 & 180 & 4,04 & Mar & 50 & 190 & 1,13 \\
\hline Abr. & 160 & 195 & 3,51 & Abr. & 150 & 200 & 3,21 \\
\hline Mai & 190 & 200 & 4,07 & Mai & 130 & 210 & 2,65 \\
\hline Jun. & 150 & 200 & 3,21 & Jun. & 140 & 210 & 2,85 \\
\hline Jul. & 180 & 190 & 4,05 & Jul. & 120 & 210 & 2,45 \\
\hline Ago. & 185 & 195 & 4,06 & Ago. & 100 & 200 & 2,14 \\
\hline Set & 190 & 190 & 4,28 & Set & 100 & 205 & 2,09 \\
\hline Out & 150 & 200 & 3,21 & Out & 225 & 220 & 4,38 \\
\hline Nov. & 210 & 200 & 4,49 & Nov. & 200 & 230 & 3,72 \\
\hline Dez & 190 & 210 & 3,87 & Dez & 210 & 240 & 3,75 \\
\hline
\end{tabular}

Fonte: os autores, 2021

Como podemos verificar no gráfico 6, a cobertura de stock teve uma diminuição positiva do ano de 2019 para 2020, isto é, em 2020, em média o stock existente permanecia parado entre 2 e 3 semanas enquanto do ano 2019 o stock permanecia entre 3 e 4 semanas. Existiu uma pequena exceção no mês de outubro de 2020 onde a permanência de stock ultrapassou as 4 semanas. 


\section{(IV) SIIEFA}

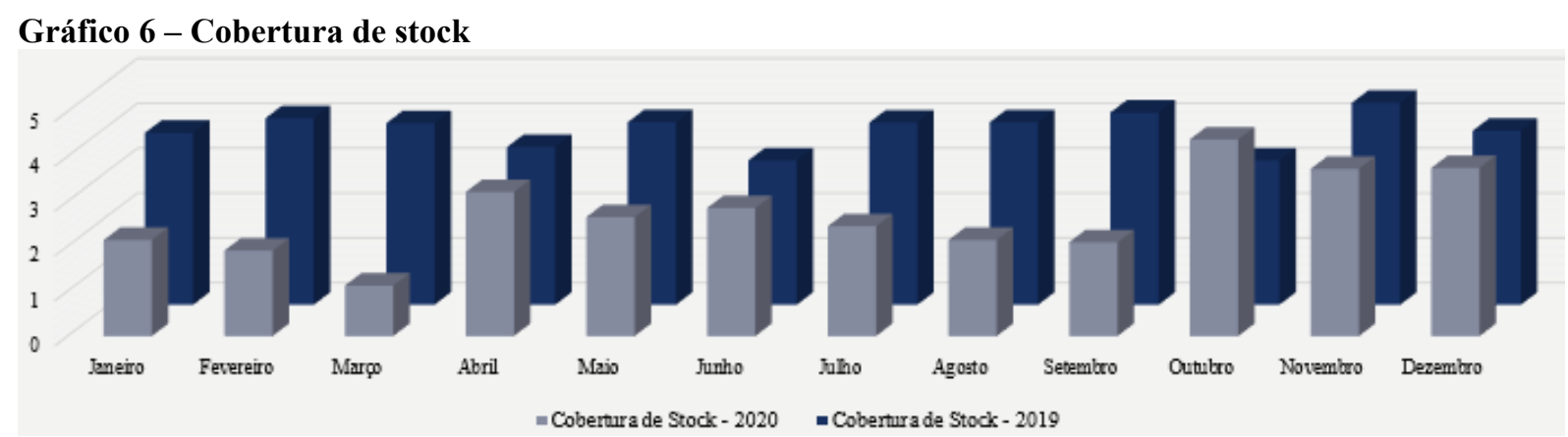

Fonte: os autores, 2021

\section{CONCLUSÃO}

O presente trabalho teve como objetivo desenvolver um roteiro para monitoramento do desempenho logístico, em uma empresa de pequeno porte, no ramo de semijoias, que atua no Centro-Oeste brasileiro, para fornecer informações que contribuam, de forma estruturada, com o gerenciamento das operações da empresa. Para isso, foi realizado um estudo de caso que comtemplou a definição dos indicadores logísticos mais adequados à empresa; e o cálculo e interpretação dos indicadores logísticos da empresa de semijoias.

Foram selecionados indicadores que fornecem informações de três perspectivas logísticas, que são entrega, transporte e stock. Os indicadores comtemplados pela perspectiva de entrega foram: a) pedidos perfeitos; b) \% de entrega no prazo; c) índice de atendimento do pedido; e d) devolução de pedidos. Na perspectiva de transporte foi considerado o custo de transporte, e na perspectiva de stock foi considerado a cobertura de stock.

Destaca-se que o horizonte de tempo considerado para o levantamento de dados contemplou os anos de 2019 e 2020. Sendo assim é importante agregar às análises e discussões à situação ocorrida em 2020, que a partir dos primeiros meses, o Brasil começou a sofrer os impactos da Pandemia por COVID 19, o que exigiu medidas de distanciamento social, apresentou grande impacto na saúde de todo o mundo, além do impacto econômico, gerando, entre outras consequências, a diminuição da renda.

No que tange aos indicadores de entrega, tem-se que todos, apesar das circunstâncias que diferenciam os cenários de 2019 e 2020, não apresentam diferença significativa entre os anos. Contudo, os indicadores de Pedido Perfeito, \% de Entregas no Prazo e Índice de Atendimento do Pedido apresentam grande variação entre os meses, com suas respectivas amplitudes, na ordem de $31 \%, 30 \%$ e $27 \%$, indicando interferência de variáveis com sensibilidade sazonal. Já o indicador de Devolução de Pedidos, aparenta ser mais estável, com amplitude de $3 \%$ (variando entre $2 \%$ e $5 \%$ ).

O indicador de transporte, Custo de Transporte, é o que apresenta maior estabilidade, apresentando 2,4\% em 2019 e 2,5\% em 2020, com amplitude mensal de $0,9 \%$.

Nesse caso, destaca-se que diante do cenário de 2020, os custos de transporte aumentaram, sendo uma das principais causas o aumento dos combustíveis, no entanto o percentual gasto com o transporte se manteve. Assim, evidencia-se que houve o repasse deste custo ao preço do produto e o volume de venda não foi impactado negativamente, o que em um cenário de crise e queda de consumo representa um crescimento da empresa, como percebido em seu faturamento.

O indicador que demonstra a perspectiva de stock, Cobertura de Stock, é o único que apresenta diferença significativa entre os valores de 2019 e 2020, sendo respectivamente 3,9 


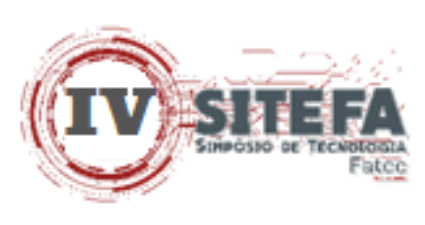

semanas e 2,7 semanas. Nesse ponto, destaca-se que a empresa melhorou seu desempenho, já que conseguiu manter as entregas para seus clientes com um volume de produtos em stock menor. Com isso, destaca-se ganhos com a redução dos riscos de avaria, além de ganhos no fluxo de caixa, considerando o menor valor de capital empregado. Salienta-se como principais hipóteses dessa melhora de desempenho o aumento da maturidade da empresa e as melhorias operacionais causadas pela pressão da pandemia.

Além dos ganhos proporcionados pela avaliação reativa dos indicadores, ou seja, por proporcionar conhecimento sobre o desempenho passado da empresa, os indicadores de desempenho logísticos podem fornecer benefícios para o planejamento e gerenciamento da empresa, fornecendo informações de modo proativo e estruturado para a tomada de decisão. Nesse ponto, destaca-se que a utilização desses indicadores para acompanhar e melhorar o desempenho operacional da empresa agregam ao processo de tomada de decisão, e consequentemente ao gerenciamento das operações da empresa, trazendo novas perspectivas para a competitividade das pequenas e médias empresas no setor de semijoias brasileiro.

No que tange às limitações do estudo, destacam-se: a) a utilização de um único caso; b) o cálculo de apenas seis indicadores; e c) a realização do estudo durante uma pandemia. Em relação à utilização de um único caso, destaca-se que proporcionou maior profundidade no estudo, e não impede a generalização em empresas do mesmo porte e setor.

Sobre o cálculo de apenas alguns indicadores, ressalta-se que não limita nem contraria os benefícios elencados com o roteiro de monitoramento, além do fato de que, para empresas menores, é melhor ter poucos (e adequados) indicadores corretamente calculados e monitorados, do que uma grande quantidade que impede seu monitoramento e gerenciamento. Já pelo fato da Pandemia, destaca-se que há influências, mas que não divergem, apenas aceleram as pressões de mercado, e o fato de ter sido considerado diferentes momentos (com e sem Pandemia) não há prejuízos para as considerações do estudo.

Por fim, como sugestão de trabalhos futuros, ressalta-se: a) realizar o estudo, para propor um roteiro de monitoramento logístico, com empresas de diferentes setores e diferentes portes, identificando a diferença de oportunidades que cada uma apresenta; b) considerar o cálculo de outros indicadores, na mesma empresa e verificar o benefício de sua interpretação e monitoramento

\section{REFERÊNCIAS}

AZEVEDO, Daniel H. Carrara. Análise e Redesenho do Processo de Suprimentos de uma Usina de açúcar e álcool: Um estudo de caso. Disponível em:

https://docplayer.com.br/351373-Analise-e-redesenho-do-processo-de-suprimentos-de-umausina-de-acucar-e-alcool-um-estudo-de-caso.html. Acesso em: 31 março 2021.

BALLOU, R.H. Gerenciamento da cadeia de suprimentos: Planejamento, organização e logistica empresarial. São Paulo: Bookman, 2001.

BANDEIRA, A. Rede de Indicadores de Desempenho para Gestão de uma Hidrelétrica. São Paulo, Dissertação (Mestrado) - Escola Politécnica, Universidade de São Paulo, 1997

CARVALHO, J. C. de. Logística e gestão da cadeia de abastecimento. 2 ed. Edições Sílabo. 2017 


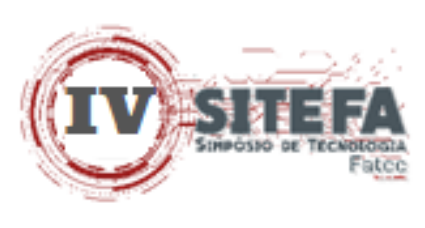

CHIAVENATO, I. Administração: teoria, processo e prática. 4. ed. Rio de Janeiro: Elsevier, 2007.

CHOPRA, S.; MEINDL, P. Supply chain managemet: strategy, planning and operation. 3. Ed. New Jersey: Pearson, 2007 não consta no trabalho, se usou tem de citar, se não usou tem de excluir daqui.

CONCEIÇÃO, S. V.; QUINTÃO, R. T. Avaliação do desempenho logístico da cadeia brasileira de refrigerantes. Revista Gestão \& Produção, São Carlos, SP, v.11, n. 3

COUNCIL of Logistics Management. What is it all about? IL: Oak Brook, 1986.

CROXTON, K.L.; GARCIA-DASTUGUE, S. J.; LAMBERT, D. The supply chain management processes. International journal of logi, 12(3), 13, 2001.

FREITAS, W. R. S.; JABBOUR, C. J. C. Utilizando estudo de caso(s) como estratégia de pesquisa qualitativa: boas práticas e sugestões. Estudo \& Debate, Lajeado, v. 18, n. 2, p. 07-22, 2011.

GANGA, Gilberto M. D. Trabalho de Conclusão de Curso (TCC) na Engenharia de Produção. São Paulo: Atlas S.A., 2012.

INSTITUTO BRASILEIRO DE GEMAS \& METAIS PRECIOSOS. O setor em grandes números. São Paulo, 2018.

LLEWELLYN, S.; NORTHCOTT, D. THE “SINGULAR VIEW” IN MANAGEMENT CASE STUDIES QUALITATIVE RESEARCH IN ORGANIZATIONS AND

MANAGEMENT. AN INTERNATIONAL JOURNAL, v. 2, n. 3, p. 194-207, 2007.

VERGARA, Sylvia Constant. Projetos e relatórios de pesquisa em administração. 5. ed. São Paulo: Atlas, 2004.

NEELY, A. et al. (1995). Performance measurement system design: A literature review and research agenda. International Journal of Production Economics. no. 4, pp 80-116.

OLAVE, Maria Elena León; AMATO NETO, João. Redes de cooperação produtiva: uma estratégia de competitividade e sobrevivência para pequenas e médias empresas. In: Gestão e Produção. v.8 n.3 São Carlos, 2001.

VOSS, C.; TSIKRIKTSIS, N.; FROHLICH, M. Case research in operations management. International Journal Of Operations \& Production Management, v. 22, n. 2, p. 195-219, 2002.

YIN, R. K. Estudo de caso - planejamento e métodos. 5.ed. Porto Alegre: Bookman, 2014. 